\title{
Hemosiderin Measurement
}

National Cancer Institute

\section{Source}

National Cancer Institute. Hemosiderin Measurement. NCI Thesaurus. Code C96659.

The determination of the amount of hemosiderin present in a sample. 